\title{
Systemic effects of fluoroscopically guided epidural steroid injection with dexamethasone
}

\author{
Woo Young Kang ${ }^{1,2}$, Joon Woo Lee ${ }^{1}$, Eugene Lee ${ }^{1}$, Yusuhn Kang ${ }^{1}$, Joong Mo Ahn', and Heung Sik Kang ${ }^{1}$ \\ ${ }^{1}$ Department of Radiology, Seoul National University Bundang Hospital, Seongnam, Korea \\ ${ }^{2}$ Department of Radiology, Chungbuk National University Hospital, Cheongju, Korea
}

Background: Epidural steroid injections (ESIs) have been widely used in managing spinal pain. Dexamethasone has recently emerged as a useful drug in this setting, relative to particulate steroids, although the associated systemic effects have not been fully elucidated. This study aimed to investigate the incidences and types of systemic effects after fluoroscopically guided ESI with dexamethasone.

Methods: This retrospective study included 888 ESIs with dexamethasone (fluoroscopically guided at the cervical and lumbosacral levels) performed on 825 patients during January to June 2017. Data regarding systemic effects were collected via telephone interviews using a standardized questionnaire at 2 weeks after the procedure. Data on patient demographic, clinical, and procedural characteristics were collected and analyzed to identify factors that were associated with systemic effects. All statistical analyses were performed using the chi-squared test.

Results: Among the 825 patients, 40 patients (4.8\%) experienced systemic effects during the 2-week follow-up period. The most common systemic effect was facial flushing (12 patients, 1.5\%), which was followed by urticaria (7 patients, $0.8 \%)$ and insomnia (7 patients, $0.8 \%)$. A history of spine surgery was significantly associated with the occurrence of systemic effects $(P=0.036)$. Systemic effects were significantly more common for injections at the cervical level than at the lumbar level $(P=0.019)$.

Conclusions: Approximately $4.8 \%$ of the patients who underwent ESI with dexamethasone experienced minor and transient systemic effects. These effects were more common in patients who had undergone a previous spine surgery or received a cervical ESI. (Korean J Pain 2019; 32: 178-86)

Key Words: Complications; Dexamethasone; Drug-Related Side Effects and Adverse Reactions; Epidural Space; Fluoroscopy; Incidence; Low Back Pain; Steroids.

Received May 8, 2019. Revised June 14, 2019. Accepted June 17, 2019.

Correspondence to: Joon Woo Lee

Department of Radiology, Seoul National University Bundang Hospital, Seoul National University College of Medicine, 82 Gumi-ro 173beon-gil, Bundang-gu, Seongnam 13620, Korea

Tel: +82-31-787-7609, Fax: +82-31-787-4011, E-mail: joonwoo2@gmail.com

ORCID: https://orcid.org/0000-0002-7106-5229

(a) This is an open-access article distributed under the terms of the Creative Commons Attribution Non-Commercial License (http:// creativecommons.org/licenses/by-nc/4.0/), which permits unrestricted non-commercial use, distribution, and reproduction in any medium, provided the original work is properly cited.

(C) The Korean Pain Society, 2019 


\section{INTRODUCTION}

Epidural steroid injections (ESIs) have been widely used in managing spinal pain caused by herniated intervertebral discs or spinal canal stenosis [1]. ESI is used to deposit steroids into the epidural space around the nerve root to manage inflammation and alleviate symptoms [2-4]. Although ESIs are increasingly used as an effective treatment for spinal pain, there continues to be debate regarding their safety and efficacy [5,6]. For example, in 2014, the US Food and Drug Administration warned that it could not confirm the safety of steroid use in the epidural space. Furthermore, particulate steroids, such as triamcinolone and methyl prednisolone, are presumed to be associated with permanent blindness, paralysis, and death after ESIs [7-10]. Thus, there are some advocates for using dexamethasone, which is a non-particulate preparation, as a replacement for particulate steroids in ESI.

A variety of systemic effects have been reported after ESI, including facial flushing, headache, vasovagal reaction, mood change, gastrointestinal problems, and cardiovascular symptoms [7-16]. However, previous studies have used varying steroids and doses, as well as different follow-up periods. Moreover, dexamethasone has a relatively short duration of use, and any associated adverse reactions are not fully elucidated. El Abd et al. [17] prospectively evaluated the adverse effects of dexamethasone use during transforaminal ESI, with 31 of 150 patients (20.7\%) experiencing adverse effects immediately after the procedure and 2 patients (1.3\%) experiencing adverse effects after 2 weeks. Nevertheless, that study only examined a single procedure (transforaminal ESI) and the sample was too small to identify rare systemic reactions. At our radiology department, all patients who undergo ESI are followed-up via telephone with a standard questionnaire to investigate the systemic effects associated with ESI at 2 weeks after the procedure. This protocol is aimed to help identify any minor systemic reactions that may occur after the procedure. Therefore, the present study aimed to investigate the incidences and types of systemic effects following fluoroscopically guided ESI with dexamethasone, as well as the relationships between the systemic effects and the patients' demographic and clinical factors.

\section{MATERIALS AND METHODS}

\section{Patients}

This retrospective observational study's protocol was approved by the Institutional Review Board of Seoul National University Bundang Hospital (No. B-1712-441-105), which waived the requirement for informed consent. During January to June 2017, 1,463 consecutive patients underwent spinal steroid injections at our department. The indications for ESI were: 1) neck/back pain or radiating pain with or without claudication, 2) nerve root compression or spinal canal stenosis identified using computed tomography or magnetic resonance imaging, and 3) failure of medical and physical therapy for $>2$ weeks. The inclusion criteria for the present study were: 1) cervical, thoracic, or lumbar ESI performed with dexamethasone via the interlaminar, transforaminal, or caudal approach and 2) completion of the follow-up telephone interview at 2 weeks after the procedure. The exclusion criteria were: 1) lack of response to the follow-up telephone interview ( $\mathrm{n}=360), 2)$ non-ESI steroid injections (e.g., facet joint injection) $(n=255)$, and 3) ESI in which the dexamethasone was administered at one-half the normal dose $(n=23)$.

\section{ESI technique}

All ESIs were performed by one of two radiologists who were experts in spinal interventions (14 years and 6 years of experience, respectively) or by trainees (fellows and residents) under the supervision of the attending radiologists. The procedure was performed under fluoroscopic guidance using a uniplanar digital subtraction angiography unit (Integris Allura Xper FD20; Philips, Best, The Netherlands). To access the epidural space, cervical ESIs were performed via two approaches (interlaminar or transforaminal) and lumbar ESIs were performed via three approaches (interlaminar, transforaminal, or caudal). The appropriate level and method were determined based on the patient's clinical symptoms and imaging findings.

The patient was placed in the prone position and the skin was sterilely prepared for all cervical and lumbar approaches, except the cervical transforaminal approach. For the cervical transforaminal approach, the patient was placed in the supine position and a 25-gauge spinal needle was inserted obliquely from the skin to the posterior aspect of 
the neural foramen, with contact to the superior articular process under fluoroscopic guidance. For the other cervical and lumbar approaches, a 22-gauge spinal needle was advanced to the target point, which was defined as the posterior epidural space for the cervical and lumbar interlaminar approach, the neural foramen under the pedicle for the lumbar transforaminal approach, and the midline cranial epidural space through the sacral hiatus for the lumbar caudal approach.

After placing the needle in the target site, a small amount of contrast media (Omnipaque ${ }^{\circledR} 300$ [iohexol] containing 300 mg iodine/mL; Amersham Health, Princeton, $\mathrm{NJ}$ ) was injected to fluoroscopically confirm the needle tip's position. If inadvertent vascular uptake was observed, the needle tip was repositioned until the intravascular uptake disappeared and an epidural flow pattern was identified. A mixture of the steroid and local anesthetic was then administered through extension tubing at the lumbar spine, although the steroid alone was administered at the cervical spine because of the potential risk for intravascular embolization when the anesthetic is mixed with a steroid [18]. Only $10 \mathrm{mg}$ of dexamethasone sodium phosphate (2 $\mathrm{mL}$ at $5 \mathrm{mg} / \mathrm{mL}$; Choongwae Pharma Corporation, Seoul, Korea) were used for the cervical transforaminal and interlaminar ESI. A local anesthetic agent, ropivacaine hydrochloride (Ropiva, $7.5 \mathrm{mg} / \mathrm{mL}$; Hanlim Pharm., Co., Ltd., Seoul, Korea), was administered in conjunction with steroids for the lumbar ESI, and the drug dosages according to each approach were as follows: 1) for the transforaminal approach, $10 \mathrm{mg}(2 \mathrm{~mL})$ of dexamethasone (5 $\mathrm{mg} / \mathrm{mL})$ and $3.75 \mathrm{mg}(0.5 \mathrm{~mL})$ of ropivacaine hydrochloride (7.5 mg/mL) with $1 \mathrm{~mL}$ saline; 2) for the interlaminar approach, $10 \mathrm{mg}(2 \mathrm{~mL})$ of dexamethasone $(5 \mathrm{mg} / \mathrm{mL})$ with $0.5 \mathrm{~mL}$ saline, and $7.5 \mathrm{mg}(1 \mathrm{~mL})$ of ropivacaine hydrochloride $(7.5 \mathrm{mg} / \mathrm{mL})$ with $2 \mathrm{~mL}$ saline; and 3) for the caudal approach, $10 \mathrm{mg}(2 \mathrm{~mL})$ of dexamethasone $(5 \mathrm{mg} / \mathrm{mL})$ with $3 \mathrm{~mL}$ saline, and $3.75 \mathrm{mg}(0.5 \mathrm{~mL})$ of ropivacaine hydrochloride $(7.5 \mathrm{mg} / \mathrm{mL})$ with $4.5 \mathrm{~mL}$ saline.

\section{Telephone interview and medical record review}

The patients were transferred to the recovery room and observed by clinical nurses under physician supervision before their discharge. The attending physician recorded any adverse events (e.g., severe pain, vasovagal syncope, and dural puncture) that occurred during or immediately after the procedure. The telephone interviews were conducted by one of two research assistants using the standardized questionnaire shown in Table 1 to identify systemic effects at 2 weeks after the procedure. The list of complications in this questionnaire was adapted from Lee et al.'s study [19], and patients were also asked to report any other side effects. The patients' electronic medical records were also searched to collect information regarding age, sex, medical history (hypertension, diabetes mellitus, heart disease, stroke, and allergies), history of spine surgery, and ESI injection site and injection technique.

\section{Statistical analysis}

All statistical analyses were performed using PASW software ver. 18.0 (IBM Corp., Armonk, NY). The chi-squared test was used to investigate the occurrence of adverse events according to the patients' demographic, clinical, and procedural characteristics. Differences were considered statistically significant at $P$ values of $<0.05$.

\section{RESULTS}

The present study included 888 ESIs that were performed on 825 patients (416 male patients and 409 female patients; mean age: 59.2 yr, range: 14-92 yr). Table 2 summarizes the patients' detailed demographic, clinical, and procedural characteristics. None of the patients experienced an immediate adverse event that interrupted the procedure or required intensive medical management. However, the telephone interview at 2 weeks revealed that

Table 1. Telephone Interview Questionnaires

\begin{tabular}{ll}
\hline \multicolumn{1}{c}{ Systemic effect } & \multicolumn{2}{c}{ Response } \\
\hline Facial flushing & Yes No Remarks \\
Urticaria/itching sense & Yes No Remarks \\
Dysmenorrhea & Yes No Remarks \\
Insomnia & Yes No Remarks \\
Myopathy & Yes No Remarks \\
Elevated blood sugar & Yes No Remarks \\
Cardiovascular problem (elevated blood & Yes No Remarks \\
pressure, palpitation) & \\
Gastrointestinal disturbances & Yes No Remarks \\
$\quad$ constipation, diarrhea, nausea, vomiting) & \\
Sexual dysfunction or menstrual change & Yes No Remarks \\
Others (psychiatric problems, hiccups) & Yes No Remarks
\end{tabular}


Table 2. Patient Demographic, Clinical, and Procedural Characteristics

\begin{tabular}{lc}
\hline Demographic/clinical/procedural factor & Value \\
\hline No. of patients & 825 \\
No. of epidural steroid injections & 888 \\
Age (yr) & $59.2(14-92)$ \\
Sex & \\
Male & 416 \\
Female & 409 \\
Patient history & \\
Hypertension & 262 \\
Diabetes mellitus & 103 \\
Heart disease & 15 \\
Stroke & 13 \\
Allergy & 3 \\
Pre procedure visual analogue scale score & $7.4(0-10)$ \\
Prior spinal surgery & 99 \\
Prior epidural steroid injection & 274 \\
Injection site & \\
Cervical & $271(32.8)$ \\
Lumbosacral & $552(66.9)$ \\
Cervical + lumbosacral & $2(0.2)^{\mathrm{a}}$ \\
Injection methods & \\
Interlaminar & $414(50.2)$ \\
Transforaminal & $311(37.7)$ \\
Caudal & $91(11.0)$ \\
More than two methods & $9(1.1)^{\mathrm{b}}$ \\
\hline
\end{tabular}

Values are presented as number only, median (range), or number (\%). ${ }^{a}$ Cervical interlaminar + lumbar transforaminal.

${ }^{b} 6$ Lumber interlaminar + transforaminal; 2 cervical interlaminar + lumbar transforaminal; 1 cervical interlaminar + transforaminal.

59 patients (59/825, 7.2\%) who underwent 62 ESIs (62/888, $7.0 \%)$ reported experiencing adverse effects; the most common event was pain exacerbation (19/825, 2.3\%), and the 40 other patients $(40 / 825,4.8 \%)$ experienced various systemic effects (Table 3). Most patients only experienced one type of systemic effect $(92.5 \%, 37 / 40)$, although $3 \mathrm{pa}-$ tients experienced two types of systemic effects $(7.5 \%$, $3 / 40$ ). The most common systemic effect was facial flushing in 12 patients (1.5\%), which was followed by urticaria (7 patients, $0.8 \%$ ), insomnia (7 patients, $0.8 \%$ ), and systemic edema (4 patients, $0.5 \%$ ). These systemic effects were transient and minor, and no patient experienced serious complications that required hospitalization.

Table 4 summarizes the relationships between systemic effects and the patients' demographic, clinical, and procedural characteristics. The patients were dichotomized at 60 years based on the mean age (59.2 yr), although
Table 3. Incidences of Systemic Effects

\begin{tabular}{lr}
\hline \multicolumn{1}{c}{ Systemic effect } & Number (\%) \\
\hline Facial flushing & $12(1.5)$ \\
Urticaria & $7(0.8)$ \\
Insomnia & $7(0.8)$ \\
Systemic edema & $4(0.5)$ \\
Elevated blood sugar & $3(0.4)$ \\
Gastrointestinal symptoms & $3(0.4)$ \\
Cardiovascular problem & $2(0.2)$ \\
Psychiatric problems & $2(0.2)$ \\
Urinary incontinence & $1(0.1)$ \\
Gynecomastia & $1(0.1)$ \\
Hiccups & $1(0.1)$ \\
Myopathy & $1(0.1)$ \\
Total & $40(4.8)$
\end{tabular}

there was no statistically significant difference in the overall incidence of systemic effects among patients who were $\geq 60$ years old and $<60$ years old $(P>0.05)$. Sex, history of hypertension, diabetes mellitus, and prior injection within 6 months were not significantly associated with the occurrence of systemic effects $(P>0.05)$. Systemic effects were more frequently observed in patients who had previously undergone spine surgery (9/99, 9.1\%) than in patients who had never undergone spine surgery (31/726, 4.3\%; $P=0.036$ ). The most frequent events among patients who had previously undergone spine surgery were facial flushing $(3 / 99,3.0 \%)$ and urticaria (3/99, $3.0 \%)$. Systemic effects were more frequent for ESIs at the cervical level $(20 / 271,7.4 \%)$ than at the lumbar level (20/552, 3.6\%; $P=0.019)$ (Table 4). The most frequent symptoms associated with the cervical spine ESI were facial flushing $(7 / 271,2.6 \%)$ and urticaria (3/271, 1.1\%). The most frequent symptoms associated with the lumbar spine ESI were facial flushing (5/552, $0.9 \%)$ and insomnia (5/552, $0.9 \%$ ) (Table 5). There was no significant difference in the overall incidence of systemic effects according to the injection method $(P>0.05)$.

\section{DISCUSSION}

This retrospective study of fluoroscopically guided ESI with dexamethasone revealed no major complications and various minor systemic effects. The overall incidence of systemic effects was low (4.8\%) and these systemic effects were most commonly encountered in patients who had 
Table 4. Relationships of Demographic, Clinical, and Procedural Characteristics with Systemic Effects

\begin{tabular}{|c|c|c|c|}
\hline \multirow{2}{*}{ Variable } & \multicolumn{2}{|c|}{ Systemic effect } & \multirow{2}{*}{$P$ value } \\
\hline & Yes & No & \\
\hline Age (yr) & & & 0.353 \\
\hline$<60$ & $16(16 / 389,4.1)$ & $373(373 / 389,95.9)$ & \\
\hline$\geq 60$ & $24(24 / 436,5.5)$ & $412(412 / 436,94.5)$ & \\
\hline Sex & & & 0.553 \\
\hline Male & $22(22 / 416,5.3)$ & $394(394 / 416,94.7)$ & \\
\hline Female & $18(18 / 409,4.4)$ & $391(391 / 409,95.6)$ & \\
\hline Hypertension & & & 0.139 \\
\hline Yes & $17(17 / 262,6.5)$ & $245(245 / 262,93.5)$ & \\
\hline No & $23(23 / 560,4.1)$ & $537(537 / 560,95.9)$ & \\
\hline Diabetes mellitus & & & 0.624 \\
\hline Yes & $6(6 / 103,5.8)$ & $97(97 / 103,94.2)$ & \\
\hline No & $34(34 / 721,4.7)$ & $687(687 / 721,95.3)$ & \\
\hline Prior injection $(<6 \mathrm{mo})$ & & & 0.806 \\
\hline Yes & $14(14 / 274,5.1)$ & $260(260 / 274,94.9)$ & \\
\hline No & $26(26 / 551,4.7)$ & $525(525 / 551,95.3)$ & \\
\hline Prior surgery & & & 0.036 \\
\hline Yes & $9(9 / 99,9.1)$ & $90(90 / 99,90.9)$ & \\
\hline No & $31(31 / 726,4.3)$ & $695(695 / 726,95.7)$ & \\
\hline Injection site ${ }^{a}$ & & & 0.019 \\
\hline Cervical & $20(20 / 271,7.4)$ & $251(251 / 271,92.6)$ & \\
\hline Lumbar & $20(20 / 552,3.6)$ & $532(532 / 552,96.4)$ & \\
\hline Injection method ${ }^{b}$ & & & 0.561 \\
\hline Interlaminar & $23(23 / 414,5.6)$ & $391(391 / 414,94.4)$ & \\
\hline Transforaminal & $12(12 / 311,3.9)$ & $299(299 / 311,96.1)$ & \\
\hline Caudal & $4(4 / 91,4.4)$ & $87(87 / 91,95.6)$ & \\
\hline
\end{tabular}

Values are presented as subject number (number/total number, \%).

${ }^{a}$ Two patients who received both injections in the cervical spine and lumbar spine were excluded from the statistical analysis because the number was too small.

${ }^{b}$ Nine patients who were injected in more than two methods were excluded from the statistical analysis because the number was too small.

previously undergone spine surgery or received a cervical ESI. Therefore, this approach may be safe and useful for patients requiring ESI.

Dexamethasone is a non-particulate and water-soluble corticosteroid [20-23]. In addition, dexamethasone is less dense than particulate steroids, such as triamcinolone, and has a smaller particle size than red blood cells, while tending to not aggregate on microscopy [24]. These properties contribute to a reduced risk of embolic infarction after intra-arterial injections, and we are not aware of any reports regarding catastrophic neurological complications associated with ESI using dexamethasone. The watersoluble preparation is taken up by cells and is immediately available, although it has a relatively short duration of ac- tion [25]. In contrast, particulate corticosteroids, which are insoluble in water, have a prolonged effect because the active moiety is released gradually during hydrolysis by cellular esterases [26]. Dreyfuss et al. [27] found that triamcinolone was more likely to be effective than dexamethasone for cervical radicular pain, despite a lack of statistical and clinical differences.

Heterogeneous study designs may explain the variable incidences of systemic effects after ESI (0.08\%-30.6\%) [19]. Lee et al. [19] recently performed a similar study, which mainly used triamcinolone, relative to the present study using dexamethasone. Although we used dexamethasone at a slightly higher dose than the equivalent dose of triamcinolone that was used by Lee et al. [19], we 
found a relatively small number of systemic effects (Table 6). Friedly et al. [28] have reported that the corticosteroid type was associated with cortisol suppression at 3 weeks after the injection, with insoluble corticosteroids causing significantly more cortisol suppression than water-soluble

Table 5. Systemic Effects according to Injection Site

\begin{tabular}{lcc}
\hline \multicolumn{1}{c}{ Systemic effect } & $\begin{array}{c}\text { Cervical ESI } \\
(n=271)\end{array}$ & $\begin{array}{c}\text { Lumbar ESI } \\
(\mathrm{n}=552)\end{array}$ \\
\hline Facial flushing & $7(2.6)$ & $5(0.9)$ \\
Urticaria & $3(1.1)$ & $4(0.7)$ \\
Insomnia & $2(0.7)$ & $5(0.9)$ \\
Systemic edema & $2(0.7)$ & $2(0.4)$ \\
Elevated blood sugar & $2(0.7)$ & $1(0.2)$ \\
Gastrointestinal symptoms & $1(0.4)$ & $2(0.4)$ \\
Cardiovascular problem & $1(0.4)$ & $1(0.2)$ \\
Urinary incontinence & $1(0.4)$ & 0 \\
Hiccups & $1(0.4)$ & 0 \\
Myopathy & $1(0.4)$ & 0 \\
Psychiatric problems & 0 & $2(0.4)$ \\
Gynecomastia & 0 & $1(0.2)$ \\
Total & $20(7.4)$ & $20(3.6)$ \\
\hline
\end{tabular}

Values are presented as number (\%).

ESI: epidural steroid injection. forms, regardless of the dose. Thus, we suggest that the rapid systemic absorption of dexamethasone may explain its relatively immediate effect, while the sustained systemic absorption of triamcinolone may explain its relatively prolonged effect. El Abd et al. [17] also reported that 31 of 150 patients (20.6\%) experienced adverse events immediately after dexamethasone injection and that 2 patients (1.3\%) reported complications at 2 weeks after the injection,

The present study revealed that systemic effects were relatively common among patients who had undergone previous spine surgery $(9 / 99,9.1 \%, P=0.036)$, although we are not aware of any other related reports. The mechanism underlying this relationship remains unclear, although we presume that it is related to increased absorption of dexamethasone due to neovascularization after surgery.

The present study revealed a significant difference in the incidences of systemic effects according to the injection site (cervical: 20/271 [7.4\%] VS, lumbar: 20/552 [3.6\%]; $P=0.019)$. A previous study by Huston et al. [11] revealed that adverse effects were significantly more common for cervical ESI relative to lumbar ESI using betamethasone, despite the steroid dose at the lumbar spine being

Table 6. Comparison of Systemic Effects after Epidural Steroid Injection between the Current Study and a Previous Study

\begin{tabular}{|c|c|c|}
\hline Variable & Lee et al. [19] & Current study \\
\hline No. of patients & 885 & 825 \\
\hline Type of injection & $\begin{array}{l}\text { Various (cervical, thoracic lumbar/ } \\
\text { interlaminar, transforaminal, caudal) }\end{array}$ & $\begin{array}{l}\text { Various (cervical, lumbar/ } \\
\text { interlaminar, transforaminal, caudal) }\end{array}$ \\
\hline Steroid drug (dose) & $\begin{array}{l}\text { Triamcinolone } 40 \mathrm{mg}(97.7 \%) / \\
\text { dexamethasone } 10 \mathrm{mg}(2.3 \%)\end{array}$ & Dexamethasone $10 \mathrm{mg}$ \\
\hline No. (\%) of patients with systemic effects & $271(30.6)$ & $40(4.8)$ \\
\hline Facial flushing (\%) & 13.6 & 1.5 \\
\hline Urticaria $(\%)^{\mathrm{a}}$ & NA & 0.8 \\
\hline Insomnia (\%) & $N A^{b}$ & 0.8 \\
\hline Systemic edema (\%) & NA & 0.5 \\
\hline Elevated blood sugar $(\%)^{\mathrm{a}}$ & NA & 0.4 \\
\hline Gastrointestinal symptoms (\%) & 5.9 & 0.4 \\
\hline Cardiovascular problem (\%) & 5.7 & 0.2 \\
\hline Psychiatric problems (\%) & 3.9 & 0.2 \\
\hline Weight/appetite change (\%) & 7.0 & 0 \\
\hline Night sweats (\%) & 4.5 & 0 \\
\hline Headache (\%) & 4.0 & 0 \\
\hline
\end{tabular}

NA: not applicable.

${ }^{a}$ Frequency of urticaria (2.5\%) and elevated blood sugar (5.2\%) was calculated as the number of systemic effects rather than the number of patients.

${ }^{b}$ Insomnia patients were included in the patients with psychiatric problems. 
double that at the cervical spine. Injected corticosteroids are absorbed through the epidural venous plexus and can exert systemic effects, as the epidural venous drainage is via Batson's plexus, which is located in the ventral epidural space. Furthermore, the dorsal venous plexus is variable in size at the lumbar level but is generally larger at the cervical level [29]. Thus, while it is difficult to precisely explain the difference in systemic effects according to the injection site, it may be related to venous drainage based on the spine level. Ahadian et al. [30] have reported that no significant difference in efficacy was found depending on the dose of dexamethasone. This, in combination with our results, could suggest that reducing the dose of steroid at the cervical level can minimize the systemic effects while maintaining effectiveness. Further investigation is indicated to determine the optimal dose of dexamethasone for ESI according to the injection site.

Our study also revealed no significant difference in systemic effects according to the injection method. The results of Derby et al. [10] and Lee et al. [19] are consistent with our study. In contrast, McGrath et al. [15] reported that adverse reactions were significantly more common after interlaminar ESI (6.0\%) than after transforaminal ESI (2.1\%). Since patients with central stenosis or diffuse symptoms were more likely to receive interlaminar injections, they assumed that patient selection would have affected the result. Although the incidences of adverse effects were low in all injection methods, practitioners should be aware that complications can occur and exercise caution.

Facial flushing was the most common systemic effect in the present study $(12 / 825,1.5 \%)$, which is assumed to be related to an immunoglobulin-mediated mechanism, although the precise etiology remains poorly understood [31]. Karaman et al. [13] and Botwin et al. [9] have also reported facial flushing in 5 of 562 patients (0.9\%) and 4 of 322 cases $(1.2 \%)$, while Everett et al. [31] reported that facial flushing was observed in 27 of 240 cases (11\%) after the lumbar transforaminal ESI. The cause of this variability is unclear, although it might be attributable to heterogeneous study designs, steroid types, and steroid doses. Nevertheless, facial flushing tends to be a minor self-limited adverse reaction after ESI, which can be managed using antihistamine medication.

This study has several limitations. First, the retrospective design and medical record review are prone to selection bias because some minor adverse effects that are not asked in a telephone survey could have been missed. However, we attempted to accurately capture the incidence and type of systemic effects using a standardized questionnaire. Second, the systemic effect data were collected at 2 weeks after the ESI, which is too early to investigate long-term adverse effects, and future research should include a long-term follow-up. Third, the incidences of systemic effects were determined via telephone interviews, rather than via a clinical examination, which raises the possibility of a discrepancy between the actual occurrence of adverse effects and the patient's subjective selfreporting. Lastly, local anesthetics such as ropivacaine used in this study, may also cause various adverse effects as well as central nervous system and cardiac toxicity [21]. Therefore, it is difficult to distinguish whether the systemic effects are due to steroid or local anesthetic.

In conclusion, various systemic effects occurred in approximately $4.8 \%$ of patients who underwent fluoroscopically guided ESI with dexamethasone. These systemic effects were more likely in patients who had undergone prior spine surgery or cervical ESI. Thus, although the dose of the injected steroid is small and intermittent, ESIs should be carefully considered in the context of the corticosteroid's potential systemic effects. This information may be used to improve pre-treatment counseling and the consent process for patients who are undergoing ESI, and may help guide the selection of safer treatment options for $\mathrm{pa}^{-}$ tients with an elevated risk of adverse effects.

\section{ACKNOWLEDGMENTS}

This work was supported by a grant from the Seoul National University Bundang Hospital Research Fund (142017-030).

\section{CONFLICT OF INTEREST}

No potential conflict of interest relevant to this article was reported.

\section{ORCID}

Woo Young Kang, https://orcid.org/0000-0002-8507-1363 Joon Woo Lee, https://orcid.org/0000-0002-7106-5229 Eugene Lee, https://orcid.org/0000-0003-4205-2362 Yusuhn Kang, https://orcid.org/0000-0003-1838-2564 
Joong Mo Ahn, https://orcid.org/0000-0002-1157-0020 Heung Sik Kang, https://orcid.org/0000-0002-7024-388X

\section{REFERENCES}

1. Manchikanti L, Pampati V, Falco FJ, Hirsch JA. Assessment of the growth of epidural injections in the medicare population from 2000 to 2011. Pain Physician 2013; 16: E349-64.

2. McLain RF, Kapural L, Mekhail NA. Epidural steroid therapy for back and leg pain: mechanisms of action and efficacy. Spine J 2005; 5: 191-201.

3. Saal JS, Franson RC, Dobrow R, Saal JA, White $A H$, Goldthwaite N. High levels of inflammatory phospholipase A2 activity in lumbar disc herniations. Spine (Phila $\mathrm{Pa} 1976$ ) 1990; 15: 674-8.

4. Bensler S, Sutter R, Pfirrmann CW, Peterson CK. Long term outcomes from CT-guided indirect cervical nerve root blocks and their relationship to the MRI findings--a prospective Study. Eur Radiol 2015; 25: 3405-13.

5. Watts RW, Silagy CA. A meta-analysis on the efficacy of epidural corticosteroids in the treatment of sciatica. Anaesth Intensive Care 1995; 23: 564-9.

6. Epstein NE. The risks of epidural and transforaminal steroid injections in the spine: commentary and a comprehensive review of the literature. Surg Neurol Int 2013; 4(Suppl 2): S74-93.

7. Botwin KP, Baskin M, Rao S. Adverse effects of fluoroscopically guided interlaminar thoracic epidural steroid injections. Am J Phys Med Rehabil 2006; 85: 14-23.

8. Botwin KP, Castellanos R, Rao S, Hanna AF, Torres-Ramos FM, Gruber RD, et al. Complications of fluoroscopically guided interlaminar cervical epidural injections. Arch Phys Med Rehabil 2003; 84: 627-33.

9. Botwin KP, Gruber RD, Bouchlas CG, Torres-Ramos FM, Freeman TL, Slaten WK. Complications of fluoroscopically guided transforaminal lumbar epidural injections. Arch Phys Med Rehabil 2000; 81: 1045-50.

10. Derby R, Lee SH, Kim BJ, Chen Y, Seo KS. Complications following cervical epidural steroid injections by expert interventionalists in 2003. Pain Physician 2004; 7: 445-9.

11. Huston CW, Slipman CW, Garvin C. Complications and side effects of cervical and lumbosacral selective nerve root injections. Arch Phys Med Rehabil 2005; 86: 277-83.

12. Pobiel RS, Schellhas KP, Eklund JA, Golden MJ, Johnson BA, Chopra S, et al. Selective cervical nerve root blockade: prospective study of immediate and longer term complications. AJNR Am J Neuroradiol 2009; 30: 507-11.

13. Karaman H, Kavak GO, Tüfek A, Yldrm ZB. The complications of transforaminal lumbar epidural steroid injections. Spine (Phila Pa 1976) 2011; 36: E819-24.

14. Plastaras C, McCormick ZL, Garvan C, Macron D, Joshi A,
Chimes G, et al. Adverse events associated with fluoroscopically guided lumbosacral transforaminal epidural steroid injections. Spine J 2015; 15: 2157-65.

15. McGrath JM, Schaefer MP, Malkamaki DM. Incidence and characteristics of complications from epidural steroid injections. Pain Med 2011; 12: 726-31.

16. Manchikanti L, Malla Y, Wargo BW, Cash KA, Pampati V, Fellows B. A prospective evaluation of complications of 10,000 fluoroscopically directed epidural injections. Pain Physician 2012; 15: 131-40.

17. El Abd O, Amadera J, Pimentel DC, Gomba L. Immediate and acute adverse effects following transforaminal epidural steroid injections with dexamethasone. Pain Physician 2015; 18: 277-86.

18. Watkins TW, Dupre S, Coucher JR. Ropivacaine and dexamethasone: a potentially dangerous combination for therapeutic pain injections. J Med Imaging Radiat Oncol 2015; 59: 571-7.

19. Lee GY, Lee JW, Yeom JS, Kim KJ, Shin HI, Kang HS. The incidence of various types of systemic reactions related to epidural steroid injections: a prospective observational study. Korean J Radiol 2018; 19: 301-10.

20. Palmer WE. Spinal injections for pain management. Radiology 2016; 281: 669-88.

21. MacMahon PJ, Huang AJ, Palmer WE. Spine injectables: what is the safest cocktail? Am J Roentgenol 2016; 207: 526-33.

22. Schneider B, Zheng P, Mattie R, Kennedy DJ. Safety of epidural steroid injections. Expert Opin Drug Saf 2016; 15: 1031-9.

23. Goodman BS, Posecion LW, Mallempati S, Bayazitoglu M. Complications and pitfalls of lumbar interlaminar and transforaminal epidural injections. Curr Rev Musculoskelet Med 2008; 1: 212-22.

24. Derby R, Lee SH, Date ES, Lee JH, Lee CH. Size and aggregation of corticosteroids used for epidural injections. Pain Med 2008; 9: 227-34.

25. MacMahon PJ, Eustace SJ, Kavanagh EC. Injectable corticosteroid and local anesthetic preparations: a review for radiologists. Radiology 2009; 252: 647-61.

26. Wright JM, Cowper JJ, Page Thomas DP, Knight CG. The hydrolysis of cortisol 21-esters by a homogenate of inflamed rabbit synovium and by rheumatoid synovial fluid. Clin Exp Rheumatol 1983; 1: 137-41.

27. Dreyfuss P, Baker R, Bogduk N. Comparative effectiveness of cervical transforaminal injections with particulate and nonparticulate corticosteroid preparations for cervical radicular pain. Pain Med 2006; 7: 237-42.

28. Friedly JL, Comstock BA, Heagerty PJ, Bauer Z, Rothman MS, Suri P, et al. Systemic effects of epidural steroid injections for spinal stenosis. Pain 2018; 159: 876-83.

29. Gala FB, Aswani Y. Imaging in spinal posterior epidural space 
186

lesions: a pictorial essay. Indian J Radiol Imaging 2016; 26 : 299-315.

30. Ahadian FM, McGreevy K, Schulteis G. Lumbar transforaminal epidural dexamethasone: a prospective, randomized, doubleblind, dose-response trial. Reg Anesth Pain Med 2011; 36:
$572-8$

31. Everett CR, Baskin MN, Speech D, Novoseletsky D, Patel R. Flushing as a side effect following lumbar transforaminal epidural steroid injection. Pain Physician 2004; 7: 427-9. 